Théorie du lien rituel anthropologie et communication de Pascal Lardellier, Paris, L'Harmattan, coll. Communication, 2003, 238 pages, $19 €$

\title{
Élizabeth Gardère
}

\section{(2) OpenEdition}

Journals

Édition électronique

URL : http://journals.openedition.org/communicationorganisation/2801

DOI : 10.4000/communicationorganisation.2801

ISSN : $1775-3546$

Éditeur

Presses universitaires de Bordeaux

Édition imprimée

Date de publication : 1 novembre 2002

ISSN : 1168-5549

Référence électronique

Élizabeth Gardère, «Théorie du lien rituel anthropologie et communication de Pascal Lardellier, Paris,

L'Harmattan, coll. Communication, 2003, 238 pages, $19 €$ », Communication et organisation [En ligne],

22 | 2002, mis en ligne le 27 mars 2012, consulté le 19 avril 2019. URL : http://

journals.openedition.org/communicationorganisation/2801; DOI : 10.4000/

communicationorganisation.2801

Ce document a été généré automatiquement le 19 avril 2019.

(c) Presses universitaires de Bordeaux 


\title{
Théorie du lien rituel anthropologie et communication de Pascal Lardellier, Paris, L'Harmattan, coll. Communication, 2003, 238 pages, $19 €$
}

\author{
Élizabeth Gardère
}

1 Rendre compte d'une théorie du lien rituel est traditionnellement un objet d'étude anthropologique. Pascal Lardellier développe une approche originale par les concepts abordés et ambitieuse par l'ouverture du spectre rituel: l'objectif théorique est de rapprocher l'anthropologie des sciences de l'information et de la communication. Dans cette perspective, la problématique centrale de l'ouvrage reconsidère les grands rites communautaires sous l'angle communicationnel. Il importe alors de centrer l'attention sur les «rites d'interactions» (E. Goffman. 1922-1982) socialement et culturellement intégrés afin de les faire passer de l'échelle interpersonnelle à un niveau communautaire.

2 L'analyse des rites communautaires mobilise un appareil conceptuel qui fait prévaloir les notions de médiation symbolique et de communication sociale. Elle se prolonge par une étude sur la dimension médiatique de leur acceptation rituelle, singularisant ainsi les formes, le sens et la fonction des rites dans les sociétés contemporaines. Parmi les auteurs à qui l'on doit un héritage théorique. Balandier, Durkheim, Mauss, Katz et Dayan sont convoqués pour asseoir ce travail et permettre à Pascal Lardellier d'élargir certaines notions de l'Ecole de Palo Alto et du courant interactionniste.

3 Au-delà d'un panorama conceptuel, il s'agit d'une réflexion sur « le dispositif rituel qui ne vaut qu'en tant qu'il devient contexte, c'est-à-dire espace-temps performatif d'un point de vue social et institutionnel, transformant les acteurs en termes d'appartenance, de changement de statut, de légitimation... ». L'apparence, souvent somptueuse, exerce alors une action d'idéalisation systématique en contribuant à rendre le rituel spectaculaire sur un modèle spéculaire : elle « donne des objets à voir, des corps à regarder ». En accédant à 
l'état d'idéalité et de complétude, degré symptomatique du dispositif rituel, le principe de présentation de Soi est sublimé au profit de la « représentation du Corps collectif ». Cette image idéale de la communauté rassemblée fait l'expérience du regard collectif et fonde un mode de communion rituelle. La puissance du rite prend toute son ampleur en offrant au spectateur un "appar-être miraculeux » dont l'efficacité est plus symbolique que mystique. Entre autres exemples, l'organisation du déroulement des rites et leur consignation (livres, protocoles, rituels, etc.) sont garants de la légitimité et de la pérennité du rite. Le contexte rituel œuvre à déconstruire et à dé-fonctionnaliser le rapport coutumier à l'espace-temps de sorte à permettre leur ré-appropriation en faveur d'un dispositif technique spécifique. Dispositif dans lequel les participants jouent un rôle précis mais ambivalent «en vertu de la double contrainte rituelle». C'est en cela que le contexte rituel est performatif.

$4 \mathrm{Si}$, comme le suggère Pascal Lardellier, "la société est intrinsèquement rituelle ", alors les effets spectaculaires de la mise en scène collective du rite ne sont que la partie visible des représentations de la vie sociale. Un retour à la nature même des symboles du lien rituel donne tout son sens aux relations entre le don et le rituel. La restauration de la fonction identitaire est ici l'une des réponses apportée d'autant qu'elle « a pour particularité de ne pouvoir être satisfaite qu'en sortant du registre de la fonctionnalité et de l'utilité ». En effet les fonctions sociales et matérielles d'un individu et d'une société ne sauraient exister sans la prise en considération du sujet en tant que tel.

5 En proposant une théorie communicationnelle du lien rituel, Pascal Lardellier s'engage sur la voix de l'interdisciplinarité avec le projet de contribuer à pallier l'«hypocondrie épistémologique » qui affecte, selon Clifford Geertz. les sciences humaines et sociales.

6 Le premier chapitre "Pluralité de l'anthropologie, permanence des médiations symboliques" retrace les évolutions et les dérives épistémologiques qui ont traversé l'anthropologie et permet à l'auteur de prendre appui sur cette discipline. En effet, «l'anthropologie étudie indirectement [...] les faits de communication, dès lors qu elle prèle attention [...] aux interactions. [...] aux représentations propres aux cultures [...].

7 Donc toute anthropologie, dès lors qu'elle étudie les formes symboliques ou techniques du lien social, étudie, peu ou prou, la communication».

8 «Le rite, un objet scientifique total» est analysé en deuxième partie. Le débat est ouvert et rebondit à l'occasion de questions, notamment sur l'utilité du rite et sur ses modes de célébration. De ces représentations sublimées, la dimension performative se distingue comme étant le trait dominant des pratiques rituelles. Régimes rituels qui, au détour d'une typologie et d'exemples de la scène politique ou médiatique sur fond historique, apparaissent en tant que dispositifs et contextes symboliques.

9 Le troisième chapitre « Rite, médiation, communication » ouvre l'approche anthropologique à la dimension communicationnelle. Le rite est ici posé comme principe de médiation. Il produit les conditions d'existence d'une communauté pour qui les notions d'espacetemps sont symbolisées en fonction de la singularité du rite.

10 La mise en regard des «rites et médias» démontre le rapport pragmatique entretenu au niveau des effets symboliques, institutionnels et politiques. Les médias rituels se voient conférer un rôle de légitimation. Vecteurs d'appartenance symbolique, leur efficacité passe par un besoin de renforcement du lien social. En devenant témoin et acteur de l'événement médiatisé, le spectateur participe de la construction symbolique d'une communauté rituelle qui régénère le moment historique. 
11 La «théorie du lien rituel» bâtit en dernière partie, rend compte des contextes de communication sociaux et formels et met l'accent sur le "principe de magnificence » (Thomas d'Aquin) des rites. Véritable théâtre du spectaculaire, de l'apparence et du pouvoir, les représentations rituelles fascinent les témoins et produisent des effets de dissociation de la perception.

12 Alain Caillé conclut l'ouvrage de Pascal Lardellier par la rédaction d'une postface qui pose, dans les actes, la question du lien rituel. En « sacrifiant bien à un impératif rituel ", ne s'agit-il pas là de reconnaitre le pouvoir symbolique d'un rite communautaire? 